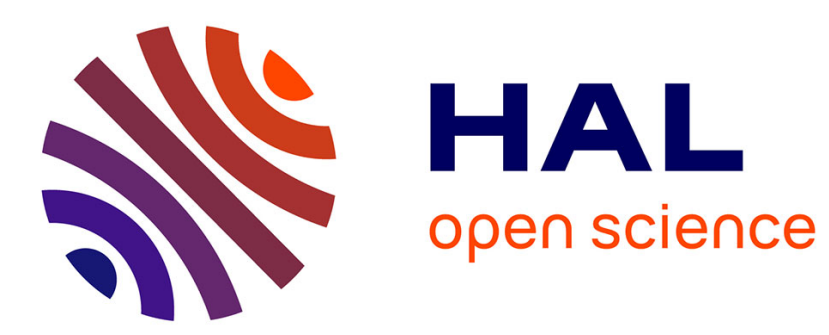

\title{
A macroscopic single-lane roundabout model to account for insertion delays and O-D patterns
}

\author{
E. Chevallier, L. Leclercq
}

\section{To cite this version:}

E. Chevallier, L. Leclercq. A macroscopic single-lane roundabout model to account for insertion delays and O-D patterns. Computer-Aided Civil and Infrastructure Engineering, 2008, Vol23,n2, p104-115. hal-00506349

\section{HAL Id: hal-00506349 \\ https://hal.science/hal-00506349}

Submitted on 27 Jul 2010

HAL is a multi-disciplinary open access archive for the deposit and dissemination of scientific research documents, whether they are published or not. The documents may come from teaching and research institutions in France or abroad, or from public or private research centers.
L'archive ouverte pluridisciplinaire HAL, est destinée au dépôt et à la diffusion de documents scientifiques de niveau recherche, publiés ou non, émanant des établissements d'enseignement et de recherche français ou étrangers, des laboratoires publics ou privés. 


\title{
A MACROSCOPIC SINGLE-LANE ROUNDABOUT MODEL TO ACCOUNT FOR INSERTION DELAYS AND O-D PATTERNS
}

\author{
Estelle Chevallier, Ludovic Leclercq ${ }^{*}$ \\ Laboratoire Ingénierie Circulation Transport \\ LICIT - ENTPE / INRETS - Université de Lyon \\ Rue Maurice Audin, 69518 Vaulx en Velin Cedex, France
}

\begin{abstract}
Available traffic simulation tools for roundabouts are exclusively based on a microscopic framework. This article provides a parsimonious macroscopic model able to replicate the main traffic features occurring at this kind of intersections. Especially, it accounts for time-limited disruptions on the approach legs due to the insertion process, even when the entry demand does not exceed the merging capacity. It also computes circulating flows which are consistent with vehicle paths induced by the O-D traffic composition. The two-step validation process using data collected at two different roundabouts is convincing. Moreover, the model appears to be computer-efficient and has few easy-to-calibrate parameters. As a result, it can be easily linked to a traffic simulation package in order to model a whole urban network.
\end{abstract}

\section{INTRODUCTION}

For few years, modelling traffic at roundabouts has become a challenging task for improving design, performance and environmental analysis. To the authors' knowledge, all existing simulation tools for this kind of intersections are based on a microscopic representation of traffic flow. The most common models listed by the Federal Highway

\footnotetext{
* to whom correspondence should be addressed.

Email: leclercq@entpe.fr

Tel: +33472047716 / Fax: +33472047712
} 
Administration (Robinson, 2000), VISSIM, PARAMICS, INTEGRATION, CORSIM or SIMTRAFFIC only differ in (i) gap-acceptance rules for modelling the insertion process and (ii) car-following rules for modelling vehicle trajectories on the circulatory roadway and on the approach and departure legs. These microscopic models are useful for a detailed modeling of vehicle behaviors, especially when different driving characteristics or vehicle types should be distinguished. However, concerns are often expressed regarding their misuse (Akçelik and Besley, 2001). Firstly, these models need lots of behavioral parameters which may be difficult to calibrate. Secondly, their stochastic nature requires several simulation runs for obtaining representative results. Consequently, it is not straightforward to incorporate microscopic roundabout models into traffic flow simulation packages for modelling large complex urban networks. On the contrary, macroscopic approaches have fewer parameters which can be easily calibrated from traffic observations and require less extensive preliminary on-field studies to specify the input data. They also reduced the burden of calculation time and computer storage which makes them well-suited for large-scale applications. Therefore, disposing of both microscopic and macroscopic models for roundabouts is appealing since it allows practitioners to select the most appropriate approach to given modelling goals and constraints.

Consequently, this paper will propose a model to fill the shortage of macroscopic simulation tools for single-lane roundabouts. This model should fulfill the following requirements:

(a) being consistent with downstream traffic conditions,

(b) being consistent with the origin-destination (O-D) patterns of entry flows,

(c) simulating accurate delays and queues caused by vehicle interactions at the entries whether the roundabout is congested or not. 
To agree condition (a) the single-lane roundabout model should satisfy the FIFO condition at the exits as presented in the Newell's diverge model (Newell, 1993b). This rule implies that, if a departure leg is congested, the congestion should spill back to the circulatory roadway. Indeed, on a one-lane roundabout, vehicles wishing to join the congested leg block the circulating ones.

One of the outcomes of (b) is that each simulated exit volume should be proportional to the sum of all entry flows with respect to the destination ratios. This is the premise of classical diverge models which split the circulating flow at the exits according to turning proportions. However, due to the looping nature of roundabouts, the origin of exiting vehicles also matters. Indeed, the O-D patterns influence vehicle trajectories inside the roundabout and, therefore, modify the circulating volume. As the circulating volume is the main impeding factor for insertion, neglecting some of the effects of the O-D patterns amplifies the modelling errors. To correctly reproduce vehicle paths on the circulatory roadway, a multi-destination formulation of the traffic flow model should be used. It consists in sharing each entry flow into partial flows according to all the possible destinations. Then, partial flows propagate on the circulatory roadway up to their corresponding exit with respect to the FIFO rule since the roundabout has only one circulating lane. This formulation makes the model fully consistent with the O-D traffic patterns at every point of the circulatory roadway.

Condition (c) depends on the choice and the calibration of the merge model. Classical macroscopic merge models allocate the downstream capacity amongst circulating and entering vehicles as in Daganzo (1995), Lebacque (1996), Buisson et al. (1996), Jin and Zhang (2003) or Lebacque and Khoshyaran (2005). The allocation process can be adapted to roundabout geometry thanks to analytical capacity formulae like the TRL linearregression based method (Kimber, 1980) or the gap-acceptance based methods as the HCM 
(Highway Capacity Manual, 2006) or the aaSIDRA (Akçelik, 2005) methods. However, despite efforts on calibration, all existing merge models fail to reproduce delays triggered by the insertion process when the entry demand does not exceed the merging capacity. Indeed, congestion only appears on a link when the supply is not sufficient to satisfy the demand. This has motivated the development of a new dynamic macroscopic merge model which accounts for the average effects of the give-way rule to meet condition (c) (Chevallier and Leclercq, 2007). This model will be used to simulate the insertion process at each roundabout entry.

The first part of this paper will give an insight of the overall algorithm. Firstly, the multi-destination formulation for both link and diverge modules will be mainlined. Secondly, the theoretical background of the merge model as well as its computer implementation will be exposed. The second section will present some simulation results. They will be shown to be consistent with empirical data collected (i) on an entire singlelane roundabout at two distinct day-time periods to validate the whole roundabout model and (ii) at one specific entry of a near-to-capacity roundabout to show the relevance of the merge model in terms of average queuing delays.

\section{BASIC COMPONENTS OF THE MODEL}

\subsection{Overview of the algorithm}

The roundabout is split out into approach links $\left(A_{i}\right)$, departure links $\left(D_{j}\right)$, circulatory links $\left(C_{m}\right)$ and intermediate circulatory links between a pair of approach and departure legs $\left(I_{n}\right)$. The number of approach (noted $\left.a\right)$ and departure legs (noted $d$ ) can be variable. This roundabout representation allows the modelling task to be reduced to a series of merge, link and diverge modules as presented in figure 1. 

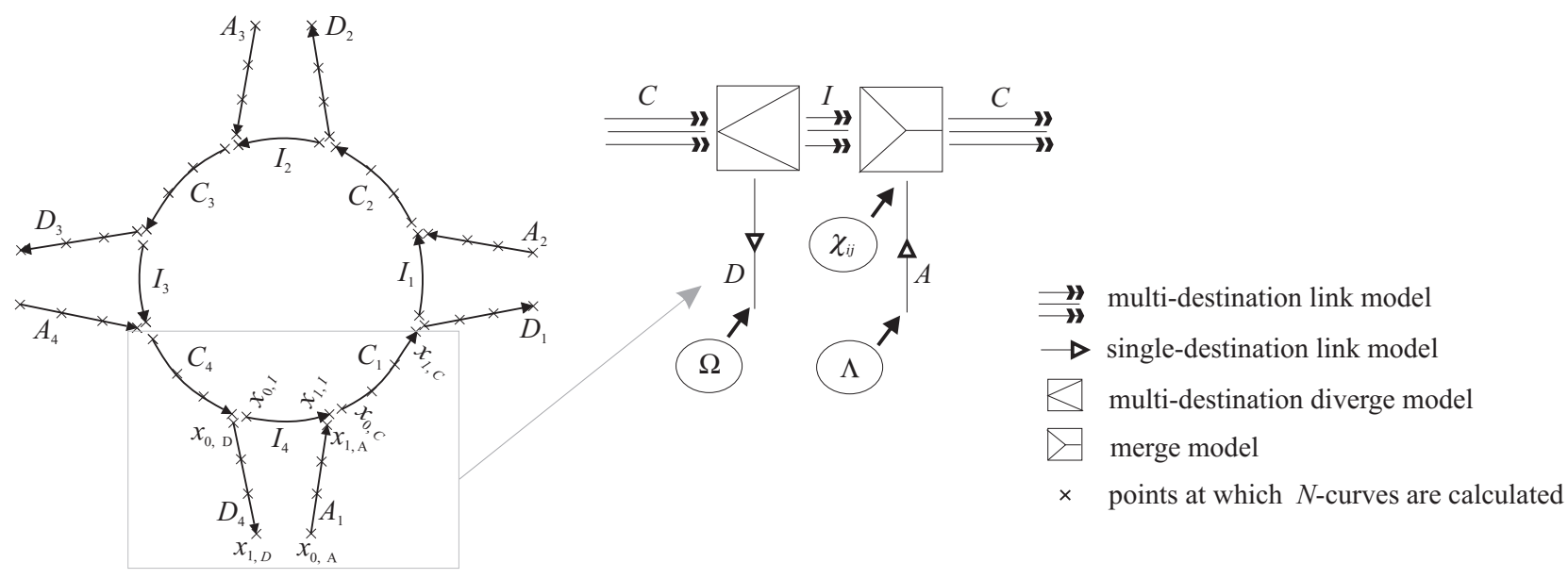

Fig.1. Roundabout modelling and conceptual framework

The exogenous inputs of the roundabout model are:

- the demand flow $\Lambda_{i}(t)$ at the beginning of each approach leg $i \in[1, a]$,

- $\quad$ the supply $\Omega_{j}(t)$ at the end of each departure leg $j \in[1, d]$,

- the O-D coefficients, $\chi_{i j}(t)$ representing the proportion of the total circulating flow which enters the roundabout at entry $i \in[1, a]$, and has to exit at leg $j \in[1, d]$.

All modules respect Newell's theory (Newell, 1993a, 1993b) in which traffic is fully described by a continuous function, $N(x, t)$, giving the cumulative vehicle count past any point $x$ by time $t$ (referred as $N$-curves in the sequel). Note that the flow $q$ can be derived from $N$ by:

$$
q=\partial_{t} N
$$

Traffic sates are described by a triangular fundamental diagram which requires only three parameters: (i) the jam density $\kappa$, (ii) the free-flow speed $u$ and (iii) the wave speed $w$ in congested regime. Note that these three parameters can vary for each stretch of road; we will add a subscript $A, D, C$ or $I$ to lift the ambiguity. The overall roundabout model includes five other parameters for calibrating the merge model which will be detailed later. 
For numerical computation, each link $L \in\{A, D, C, I\}$ is divided into cells of length $\Delta x_{L}$. The first and last cell boundaries of $L$ are noted $x_{0, L}$ and $x_{1, L}$ (see figure 1 ). $N_{L}$ denotes the $N$-curve of link $L$. It is calculated at each cell boundary of the grid at every time-step $\Delta t$. As we will see in the following section, all the $N$-curves will be updated according to values calculated at previous time-steps but not according to current time values output by other modules. As a result, the order of implementation of the modules in the overall algorithm does not matter. This is a convenient property given the looping nature of roundabouts. It allows the model to be easily integrated into a traffic flow simulation package of a whole network.

\subsection{Single-destination link model}

This module simulates traffic flow on the approach and departure legs where the multidestination formulation is not required. In this section, $L \in\{A, D\}$. For all cell boundaries except the extremities, $N$ is numerically computed according to the Newell's model (1993a) which simplifies to (2) providing a triangular fundamental diagram. This model use the concept of demand and supply defined in Daganzo (1995) and Lebacque (1996). It assumes that, at a cell boundary $x$, it is possible to update the $N$-curve at time $t$ according to (i) its value at the upstream cell boundary at the previous time-step (the demand term, representing the flow willing to advance) and (ii) the sum of its value at the downstream cell boundary at time $t-\Delta x_{L} / w_{L}$ and a fixed term $\Delta x_{L} \kappa_{L}$ (the supply term, representing the remaining capacity due to downstream congestion).

$$
N_{L}(x, t)=\min [\underbrace{N_{L}\left(x-\Delta x_{L}, t-\Delta t\right)}_{\text {demand }}, \underbrace{N_{L}\left(x+\Delta x_{L}, t-\frac{\Delta x_{L}}{w_{L}}\right)+\Delta x_{L} \kappa_{L}}_{\text {supply }}] \forall x \notin\left\{x_{0, L}, x_{1, L}\right\}
$$


Parameters and grid cells are chosen for having $\Delta x_{L} / \Delta t=u_{L}$ (Courant-Friedrich-Levy condition). Note that if $u_{L} / w_{L}$ is an integer the numerical scheme is exact (Daganzo, 2005a, 2005b). Otherwise, $N$ is interpolated between two known values which induce only slight numerical errors. For practical issue the time-step $\Delta t$ is common to all stretches of road; thus, the CFL condition imposes different grid cell sizes depending on $u_{L}$. At the first and last cell boundaries, equation (2) still applies but the demand and supply are given by either the exogenous demand (respectively supply) at the beginning (respectively at the end) of the link or the merge and diverge modules (see table 1).

\begin{tabular}{|c|c|c|c|c|}
\hline $\begin{array}{c}\text { cell } \\
\text { boundary }\end{array}$ & $x_{0, A}$ & $x_{0, D}$ & $x_{1, A}$ & \multicolumn{1}{c|}{$x_{1, D}$} \\
\hline demand & $N_{A}\left(x_{0, A}, t-\Delta t\right)+\Lambda(t) \Delta t$ & $\begin{array}{c}\text { from the } \\
\text { diverge } \\
\text { module }\end{array}$ & \multicolumn{2}{|c|}{ equation (2) } \\
\hline supply & \multicolumn{2}{|c|}{ equation (2) } & $\begin{array}{c}\text { from the } \\
\text { merge } \\
\text { model }\end{array}$ & $N_{D}\left(x_{1, D}, t-\Delta t\right)+\Omega(t) \Delta t$ \\
\hline
\end{tabular}

Tab. 1. Demand and supply for link extremities

\subsection{Multi-destination formulation}

As discussed in the introduction, the single-destination model does not account for all the effects of the O-D patterns of entry flows. This has motivated the development of the multi-destination link and diverge modules.

\subsubsection{Multi-destination link model}

The entering flow from link $A_{i}$ computed by the merge model is immediately split out into partial streams $j$, according to $\chi_{i j}(t)$. The multi-destination link module is then used to update the partial $N$-curves noted $N_{C, j}$ and $N_{I, j}$ at any points of the link except the extremities. The basic principle of this model is that, on one-lane links, the trip travel time between two cell boundaries should be the same for each partial stream because the delay 
suffered by a vehicle is independent of its destination (FIFO condition). The algorithm is presented for the $C$-type links but is exactly the same for the $I$-type links.

Step 1: updating $N_{C}(x, t) \forall x \notin\left\{x_{0, C}, x_{1, C}\right\}$ according to the single-destination model (2) with $L=C$.

Step 2: computing the effective travel time $T(t)$ of the total flow.

We have to search for the instant at which the value of the demand curve at the previous cell boundary $N_{C}\left(x-\Delta x_{C},:\right)$ is equal to the target value $N_{C}(x, t)$, see figure 2 . The difference between $t$ and this instant represent the travel time $T(t)$ between the two cell boundaries $x$ - $\Delta x_{C}$ and $x$.

Step 3: updating the partial $N$-curves at $x$ from their values at $x$ - $\Delta x_{C}$, considering that the travel time is the same for all destinations (see figure 2):

$$
N_{C, j}(x, t)=N_{C, j}\left(x-\Delta x_{C}, t-T(t)\right) \quad \forall x \notin\left\{x_{0, C}, x_{1, C}\right\}
$$

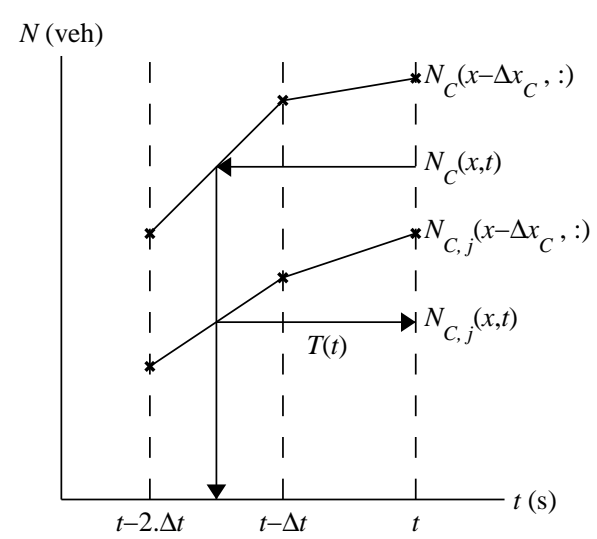

Fig.2. Computation of the travel time

\subsubsection{Multi-destination diverge model}

When partial flows reach the end of a $C$-type link they should be shared amongst the departure and intermediate links in order to feed both the single-destination and multidestination modules. This is the aim of the diverge model. Theoretical background was 
introduced by Newell (1993b) but we attempt, here, to provide a comprehensive and efficient algorithm. We note $s \in[1, d]$ the partial flow which should exit at link $D$ as depicted in figure 3.

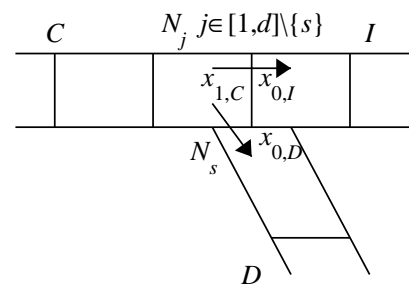

Fig.3. Notations for the diverge model

Step 1: specifying the partial streams $j \in[1, d] \backslash\{s\}$ which have to drive towards the intermediate link $I$. The partial stream $s$ should exit at link $D$.

Step 2: calculating $N_{D}\left(x_{0, D}, t\right)$ and $N_{I}\left(x_{0, I}, t\right)$ without accounting for the FIFO condition. The demand term for $N_{C}$ in equation (2) is divided according to the two possible destinations $(I$ or $D)$. Equation (2) is then applied two times with a specific supply term for each movement:

$$
\begin{gathered}
N_{D}\left(x_{0, D}, t\right)=\min \left[N_{C, s}\left(x_{1, C}-\Delta x_{C}, t-\Delta t\right) ; N_{D}\left(x_{0, D}+\Delta x_{D}, t-\frac{\Delta x_{D}}{w_{D}}\right)+\Delta x_{D} \kappa_{D}\right] \\
N_{I}\left(x_{0, I}, t\right)=\min \left[\sum_{j \in[1, d] \backslash s s\}} N_{C, j}\left(x_{1, C}-\Delta x_{C}, t-\Delta t\right) ; N_{I}\left(x_{0, I}+\Delta x_{I}, t-\frac{\Delta x_{I}}{w_{I}}\right)+\Delta x_{I} \kappa_{I}\right]
\end{gathered}
$$

Step 3: computing the expected travel time of exiting vehicles at time $t, T_{D}(t)$, for which the demand curve is $N_{C, s}\left(x_{1, C}-\Delta x_{C},:\right)$ and the target value is $N_{D}\left(x_{0, D}, t\right)$ (see section 2.3.1). 
Step 4: computing the expected travel time of all through vehicles, $T_{I}(t)$, for which the demand curve is $\sum_{j \in[1, d]\{s\}} N_{C, j}\left(x_{1, C}-\Delta x_{C},:\right)$ and the target value is $N_{I}\left(x_{0, I}, t\right)$ (see section 2.3.1).

Step 5: computing the effective travel time $T(t)$ of vehicles crossing the diverge. As each partial stream should have the same travel time:

$$
T(t)=\max \left[T_{D}(t) ; T_{I}(t)\right]
$$

Step 6: the movement having the shortest computed travel time at steps 3 or 4 should experience the same travel time as the other movement to fulfill the FIFO condition. To simplify the algorithm, we choose to update both $N_{D}$ and $N_{I}$. The updating process is similar to section 2.3.1:

$$
\begin{gathered}
N_{D}\left(x_{0, D}, t\right)=N_{C, s}\left(x_{1, C}-\Delta x_{C}, t-T(t)\right) \\
N_{I}\left(x_{0, I}, t\right)=\sum_{j \in[1, d] \backslash\{s\}} N_{C, j}\left(x_{1, C}-\Delta x_{C}, t-T(t)\right)
\end{gathered}
$$

Step 7: updating the partial $N$-curves of link $C$ for streams towards link $I$ following the same process as in step 6:

$$
N_{C, j}\left(x_{1, C}, t\right)=N_{C, j}\left(x_{1, C}-\Delta x_{C}, t-T(t)\right) \quad \forall j \in[1, d] \backslash\{s\}
$$

Step 8: updating the other partial $N$-curves of links $C$ and $I$ by remarking that $x_{0, I}, x_{0, D}$ and $x_{1, C}$ represent the same geometrical position:

$$
\begin{aligned}
& N_{C, s}\left(x_{1, C}, t\right)=N_{D}\left(x_{0, D}, t\right) \\
& N_{I, s}\left(x_{0, I}, t\right)=0 \\
& N_{I, j}\left(x_{0, I}, t\right)=N_{C, j}\left(x_{1, C}, t\right) \quad \forall j \in[1, d] \backslash\{s\}
\end{aligned}
$$

Step 9: updating the total $N$-curve of link $C$ as the sum of the partial $N$-curves: 


$$
N_{C}\left(x_{1, C}, t\right)=\sum_{j=1}^{d} N_{C, j}\left(x_{1, C}, t\right)
$$

\subsection{Merge model}

Substantially, the only missing values to implement the algorithm are the entering flow, $q_{A}$, and the circulating flow, $q_{I}$, given by the merge model from the demand levels $\lambda_{A}$ and $\lambda_{I}$ just upstream the conflict point (see figure $4 \mathrm{a}$ for notations). Then, according to equation (1), $N_{I}\left(x_{1, I}, t\right), N_{A}\left(x_{1, A}, t\right)$ and $N_{C}\left(x_{0, C}, t\right)$ can be directly updated. The merge model is based on two flow allocation schemes providing the downstream cell of the circulatory link, $C$, is congested or not. These schemes are summarized in figure $4 \mathrm{~b}$ and $4 \mathrm{c}$ and detailed below.

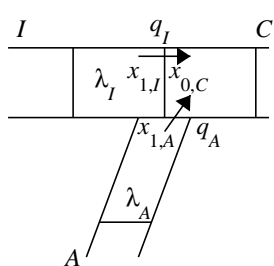

a: notations

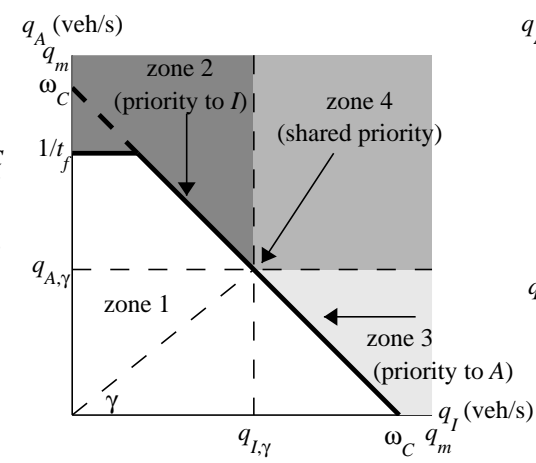

b: in congested regime

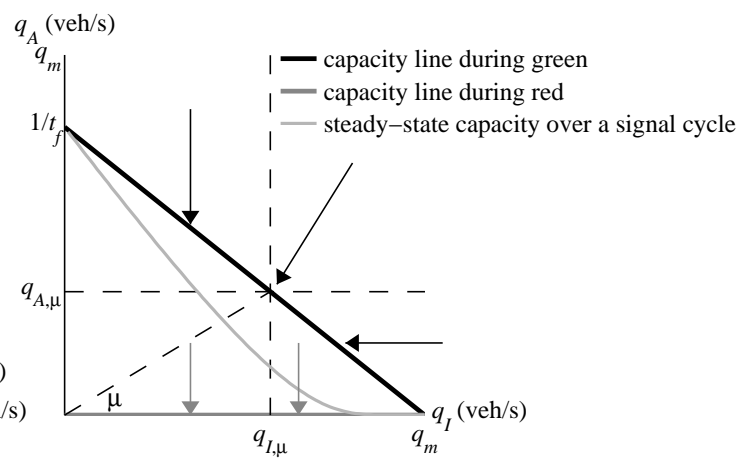

c: in free-flow regime

Fig.4. Principles of the two flow allocation schemes

\subsubsection{Model in congested regime on the circulatory roadway}

If a congestion appears on the circulatory roadway, vehicles on the upstream link $I$ tends to be bunched and drivers on the approach leg $A$ will not respect the give-way rule anymore. Instead, it could be assumed that queued vehicles from links $A$ and $I$ enter the merge in some nearly fixed congested priority ratio, noted $\gamma$, independent of the merge outflow as observed by Cassidy and Ahn (2005) on freeway on-ramps. Moreover, the lost times due to the give-way rule at the entry become negligible compared to the delays 
induced by congestion. Therefore, the allocation of the total restricted supply of the merge, $\omega_{C}$, can be done with the Daganzo's merge model (1995) which simulates accurate delay estimates when the initial demand $\left(\lambda_{I}, \lambda_{A}\right)$ lies above the capacity curve. In this model, we assume that the maximum entering flow from link $A$ is equal to the inverse of the follow-up time, $t_{f}$, which represents the time span between the departure of one entering vehicle and the departure of the next, under a condition of continuous queuing on the approach leg. Because of acceleration or visibility constraints during the insertion process, $1 / t_{f}$ can be lower than the circulating roadway capacity $q_{m}$. The capacity curve, giving $q_{A}$ in terms of $q_{I}$, is linear since vehicles on both roads are expected to optimize the allocation of $\omega_{C}$. It can be truncated by $1 / t_{f}$ if $1 / t_{f}<\omega_{C}$ as in figure $4 \mathrm{~b}$.

\section{Flow allocation in congested regime}

The main asset of the Daganzo's model is to account for the congested priority ratio $\gamma$. The line of slope $\gamma$, coming from the origin, intercepts the capacity curve at point $\left(q_{I, \gamma}, q_{A, \gamma}\right)$. Then, four different allocation rules of the initial demand $\left(\lambda_{I}, \lambda_{A}\right)$ are specified to find $\left(q_{I}, q_{A}\right)$ (see arrows in figure $4 \mathrm{~b}$ ):

- when $\left(\lambda_{I}, \lambda_{A}\right) \in$ zone 1 , both demand flows are satisfied: $\left(q_{I}, q_{A}\right)=\left(\lambda_{I}, \lambda_{A}\right)$,

- when $\left(\lambda_{I}, \lambda_{A}\right) \in$ zone $2, q_{I}=\lambda_{I}$ and the remaining capacity is allocated to link $A$,

- when $\left(\lambda_{I}, \lambda_{A}\right) \in$ zone $3, q_{A}=\lambda_{A}$ and the remaining capacity is allocated to link $I$,

- when $\left(\lambda_{I}, \lambda_{A}\right) \in$ zone $4, q_{I}=q_{I, \gamma}$ and $q_{A}=q_{A, \gamma}$. 


\subsubsection{Model in free-flow condition on the circulatory roadway}

The Daganzo's model is not able to reproduce the delays triggered by the give-way rule when the initial demand point $\left(\lambda_{I}, \lambda_{A}\right)$ lies below the capacity curve. As these delays prevail in uncongested regime, this can be a serious limitation to assess, for instance, the impacts of re-routing policies or of signal coordination schemes in the vicinities of the roundabout. This has motivated the development of a new merge model which is fully described in Chevallier and Leclercq (2007). We will just summarize its key components below.

The basic principle of the merge model in uncongested conditions is to express the average effects of the stochastic interactions between circulating and entering vehicles in terms of a deterministic fictive traffic light on the approach leg. To this end, the signal timing calculation is derived from classical assumptions about the gap-acceptance process:

- a deterministic insertion rule with a critical gap $t_{c}$ constant over time and common to all vehicles,

- a given density probability distribution of the time intervals between two consecutive vehicles on the circulating roadway upstream of the conflict point (termed headway), $f_{H}$.

Green and red periods $(G$ and $R$ ) are calculated to represent the average length and frequency of available and busy time periods for insertion (usually termed gaps and blocks).

The second key feature of this merge model is to account for the potential influence of entering vehicles over the circulating traffic by distinguishing two priority modes in the flow allocation process. The first one is the absolute priority mode in which manoeuvres of 
entering vehicles do not affect drivers on the roundabout. The other mode is the limited priority mode, initially introduced by Troutbeck and Kako (1999), in which the circulating vehicles were sometimes forced to slow-down to accommodate gap-forcing entering vehicles. Thus, $f_{H}$ is slightly modified when computing $G$ and $R$.

In Chevallier and Leclercq (2007) the results are presented for both priority modes and a shifted exponential distribution of the headways with location parameter $t_{m}$. This parameter represents the minimum headway and is assumed to perfectly match the inverse of $q_{m}$. Resulting green and red periods are depicted in figure 5 for $t_{c}=4.5 \mathrm{~s}$ and $t_{m}=2 \mathrm{~s}$. Reassuringly, whichever the priority mode, $G$ and $R$ only depends on the average impeding flow upstream the merge noted $\overline{q_{I}}$. Note that, for a merge, the impeding traffic corresponds to the traffic on the major road whereas, at a roundabout entry, it could encompass both the circulating traffic and a part of the exiting flow (Hagring, 2001) (Mereszczak et al., 2006). It is worth keeping in mind that the fictive traffic light has no physical existence. It is just introduced to mimic the average effects of stochastic vehicle interactions at a roundabout entry. We will see in the validation part of this paper that it allows for simulating non-zero delays when the entry demand is lower than the merging capacity, which is consistent with observations. 


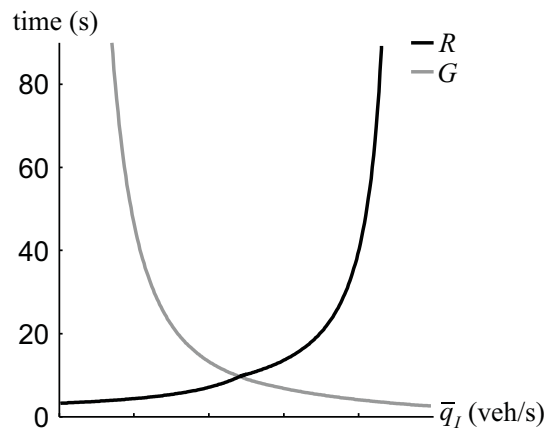

Fig. 5. Green and red periods for a shifted exponential distribution of the headways with location parameter $t_{m}=2 \mathrm{~s}$ and $t_{c}=4.5 \mathrm{~s}$

\section{Flow allocation in free-flow regime}

Contrary to the merge model in congestion, the flow allocation process varies with the signal colour (see arrows in figure 4c):

(i) during green periods the flows $q_{A}$ and $q_{I}$ are calculated according to the Daganzo's principle. Instead of $\gamma$ introduced in the congested regime we use a free-flow priority ratio, $\mu$. The couple $\left(q_{I, \mu}, q_{A, \mu}\right)$ is defined similarly to $\left(q_{I, \gamma}, q_{A, \gamma}\right)$ in the Daganzo's model but with a capacity line calibrated for roundabouts:

$$
q_{A}=\frac{1}{t_{f}}-q_{I} \frac{t_{m}}{t_{f}}
$$

In the absolute priority mode $t_{c}$ is assumed equal to $t_{f}+t_{m}$; in the limited priority mode it linearly decreases up to $t_{f}$.

Note that the switch between the absolute priority and the limited priority modes occurs when $q_{I}$ exceeds $q_{I, \mu}$. Thus, $\mu$ should be calibrate accordingly and may be not equal to $\gamma$

(ii) during red periods $q_{A}=0$ and the total outflow is allocated to link $I$. 
It should be noted that when flows are aggregated over a fictive cycle length the obtained capacity curve (referred as the steady-state capacity curve) is perfectly relevant with classical capacity formulae. This can be checked in figure 6 which compares it with:

- a classical analytical formula (see $\mathrm{Wu}, 2001$ for a review):

$$
q_{A}=\frac{q_{I} \mathrm{e}^{-\frac{q_{l}}{1-q_{I} t_{m}}\left(t_{c}-t_{m}\right)}}{1-\mathrm{e}^{-\frac{q_{I}}{1-q_{I} t_{m}} t_{f}}}
$$

- $\quad$ the Aasidra standard (Akçelik and Besley, 2004)

$$
q_{A}=\frac{1}{t_{f}} f_{o d}\left(1-t_{m} q_{I}+0.5 t_{f} \varphi q_{I}\right) e^{-\frac{q_{I}}{1-q_{I} t_{m}}\left(t_{c}-t_{m}\right)}
$$

where the O-D factor, $f_{o d}$, and the proportion of unbunched vehicles on the circulatory roadway, $\varphi$, are set equal to 1 .

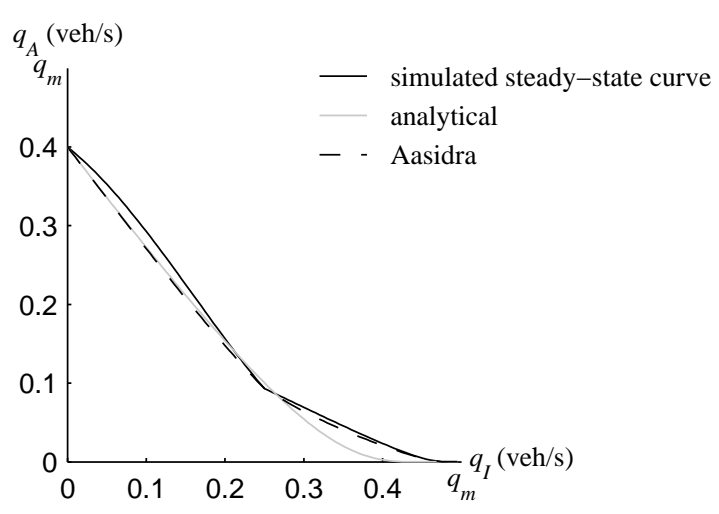

Fig. 6. Comparison of the steady-state capacity curve with standards

The performance of the merging model compared to delay and queue-length HCM standards can be found in Chevallier and Leclercq (2007).

\subsubsection{Time-scale and update of the signal-timing calculation}

An important requirement for the merge model is to be consistent with demand fluctuations on the circulatory roadway. This consistency is related to two issues in the signal timing calculation (i) at which aggregation scale should be computed $\overline{q_{I}}$ to derive 
the signal timing (referred to as the reference period) and (ii) how can be updated the green and red periods at each time-step.

\section{a. Reference period}

Extensive simulation studies were conducted to find the most appropriate reference period. It should be sufficiently long to smooth quick demand fluctuations on the circulatory roadway which are not representative of average traffic flow conditions. Conversely, it should be relatively short to ensure an optimum responsiveness of the merge model to non-trivial demand variations. After balancing these two conditions on different tested scenarios, we opted for a fixed reference period of 90s.

\section{b. $\quad$ Signal timing update scheme}

The signal timing update scheme also plays a role in the responsiveness of the merge model. Imagine that $\overline{q_{I}}$ approaches $q_{m}$ at the current time-step. The predicted red period then approaches infinity. If at the next time-steps $R$ was not updated, the traffic signal color would remain red whatever the traffic demand flow variations are. To avoid this issue, we chose a signal timing update plan in which green and red periods are re-calculated at each time-step without any memory of what happened before. This seems to be relevant with the consistency and independency assumptions regarding the drivers' insertion choice process. Moreover, only two variables should be stored: the signal color of the traffic light at the previous time-step and the period for which this color is set. Suppose that at time $t-\Delta t$ the signal colour was red for a period $\tau$. A time $t$, the signal colour switches to green only if: $R(t)-\tau<\Delta t$

Some errors can appear when $\overline{q_{I}}$ varies since $G$ and $R$ may be truncated by the update scheme. Yet, these errors are bounded and usually break-even over a simulation period. 
Note that the model can handle other signal timing update schemes keeping track of previous $G$ and $R$ values in order to limit the truncating errors.

\subsubsection{Algorithm implementation}

The previous comments on both the theoretical background and the practical implementation of the merge model are summarized below into a detailed algorithm. The merge model has finally five easy-to-calibrate parameters:

- the probability density function, $f_{H}$ of the headways in the circulating flow,

- the minimum headway, $t_{m}$,

- the follow-up time, $t_{f}$,

- the free-flow priority ratio, $\mu$,

- $\quad$ the congested priority ratio, $\gamma$.

The outputs of the model are the total and partial $N$-curves at the entry of links $A$ and $I$ which feed the multi-destination and the single-destination models. They are computed according to the following steps.

Step 1: computing the average impeding flow $\overline{q_{I}}$ as the mean of flows $q_{I}$ (and possibly a part of exiting flows $q_{D}$ ) over the last 90s-period

Step 2: determining the prevailing priority mode by comparing $\overline{q_{I}}$ and $q_{I, \mu}$

Step 3: calculating new values of green and red at time $t, G(t)$ and $R(t)$, from $\overline{q_{I}}$

Step 4: updating the signal timing according to the without memory scheme exposed in section 2.4.3.

Step 5: computing the entry demand flow $\lambda_{A}(t)$ and the circulating demand flow $\lambda_{I}(t)$ at the conflict point according to the outputs of the single-destination and multipledestination link modules at previous time-steps. As defined by Daganzo (1995) or 
Lebacque (1996), $\lambda_{L}(t)(L \in\{I, A\})$ is the minimum between the maximum flow and the flow crossing boundary $x_{1, L}$ at time $t$.

From equation (1), this flow is equal to $\frac{N_{L}\left(x_{1, L}, t\right)-N_{L}\left(x_{1, L}, t-\Delta t\right)}{\Delta t}$.

Moreover, in free-flow conditions $N_{L}\left(x_{1, L}, t\right)=N_{L}\left(x_{1, L}-\Delta x_{L}, t-\Delta t\right)$ from equation (2) so:

$$
\begin{aligned}
& \lambda_{I}(t)=\min \left[\frac{N_{I}\left(x_{1, I}-\Delta x_{I}, t-\Delta t\right)-N_{I}\left(x_{1, I}, t-\Delta t\right)}{\Delta t} ; q_{m}\right] \\
& \lambda_{A}(t)=\min \left[\frac{N_{A}\left(x_{1, A}-\Delta x_{A}, t-\Delta t\right)-N_{A}\left(x_{1, A}, t-\Delta t\right)}{\Delta t} ; q_{m}\right]
\end{aligned}
$$

Step 6: computing the flows $q_{I}$ and $q_{A}$ according to the flow allocation scheme in freeflow regime (see section 2.4.2).

Step 7: computing the downstream supply, $\omega_{C}(t)$. This is the minimum between the maximum flow and the flow crossing boundary $x_{0, C}$ at time $t$.

From equation (1), this flow is equal to $\frac{N_{C}\left(x_{0, C}, t\right)-N_{C}\left(x_{0, C}, t-\Delta t\right)}{\Delta t}$.

Moreover, in congested conditions $N_{C}\left(x_{0, C}, t\right)=N_{C}\left(x_{0, C}+\Delta x_{C}, t-\frac{\Delta x_{C}}{w_{C}}\right)+\Delta x_{C} \kappa_{C}$ from equation (2)

So:

$$
\omega_{C}(t)=\min \left[\frac{N_{C}\left(x_{0, C}+\Delta x_{C}, t-\frac{\Delta x_{C}}{w_{C}}\right)+\Delta x_{C} \kappa_{C}-N_{C}\left(x_{0, C}, t-\Delta t\right)}{\Delta t} ; q_{m}\right]
$$

Step 8: checking the traffic state on the circulatory roadway 
If $q_{I}+q_{A}>\omega_{C}, q_{I}$ and $q_{A}$ are re-calculated according to the flow allocation scheme in congested regime (see section 2.4.1).

Step 9: updating the $N$-curves of links $I, A$ and $C$ using equation (1):

$$
\begin{gathered}
N_{I}\left(x_{1, I}, t\right)=N_{I}\left(x_{1, I}, t-\Delta t\right)+q_{I} \Delta t \\
N_{A}\left(x_{1, A}, t\right)=N_{A}\left(x_{1, A}, t-\Delta t\right)+q_{A} \Delta t \\
N_{C}\left(x_{0, C}, t\right)=N_{C}\left(x_{0, C}, t-\Delta t\right)+\left(q_{A}+q_{I}\right) \Delta t
\end{gathered}
$$

Step 10: updating the partial $N$-curves of link $C$

The conservation law is applied at boundary $x_{0, C}=x_{1, I}=x_{1, A}$ :

$$
N_{C, j}\left(x_{0, C}, t\right)=N_{C, j}\left(x_{0, C}, t-\Delta t\right)+\underbrace{\chi_{i j}(t) q_{A} \Delta t}_{\begin{array}{c}
\text { additional number } \\
\text { of vehicles from } A
\end{array}}+\underbrace{N_{I, j}\left(x_{1, I}, t\right)-N_{I, j}\left(x_{1, I}, t-\Delta t\right)}_{\begin{array}{c}
\text { additional number } \\
\text { of vehicles from } I
\end{array}} \forall j \in[1, d]
$$

\section{MODEL VALIDATION}

In this section, the roundabout model is applied to on-field situations. In order to validate the overall algorithm, data were collected on a single-lane roundabout in Toulouse urban area (France) (see figure 7a). It will be shown that the model is consistent with the O-D patterns of entry flows. However, the entry and circulating flows are often too low for a complete assessment of the delays predicted by the merge model. Hence, the merge model validation was completed thanks to data collected at one conflict zone of a large roundabout in the Lyon surrounding area (France), next to a diamond exchange zone with a freeway (see figure 7b). The selected approach and departure legs are one-lane width. The roundabout has normally two circulating lanes except at the studied conflict zone where there is only one because of the presence of road pavement in front of the splitter island. Therefore, the experimental site meets the geometrical requirements to apply the merge model. 


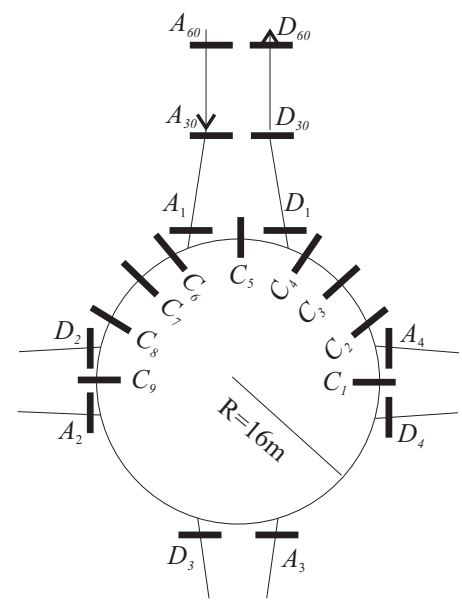

a: on a whole roundabout for the overall model validation vehicle counts

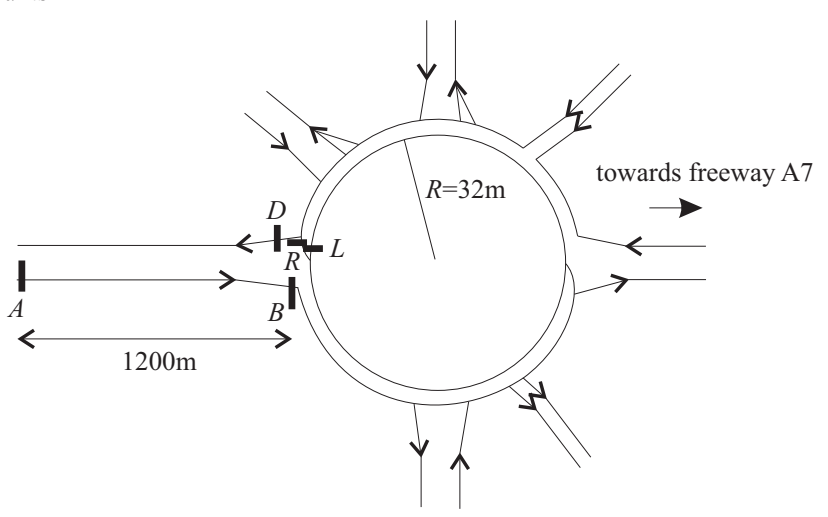

b: at a single entry for the merge model validation

\section{Fig.7. Data collection process}

\subsection{Overall model validation}

\subsubsection{Data collection}

The overall algorithm validation consists in comparing the simulated circulating and exiting flows to observations in order to check the relevance of the roundabout model. Data were collected at the single-lane roundabout illustrated in figure $7 \mathrm{a}$ during a two-hour period in the morning (8:00am to 10:00am) and a two-hour period in the afternoon (4:30pm to 6:30pm). A video camera was placed to record all vehicle movements on the roundabout. An image processing software (called AUTOSCOPE) was used to extract passing times and vehicle identities (ID) at nine different locations on the circulatory roadway (noted $C_{1}$ to $C_{9}$ ) and at about $4 \mathrm{~m}$ before each entry $\left(A_{1}\right.$ to $\left.A_{4}\right)$ and after each exit $\left(D_{1}\right.$ to $\left.D_{4}\right)$. For the approach leg 1 two additional positions were analysed at respectively $30 \mathrm{~m}$ and $60 \mathrm{~m}$ from the yield-line $\left(A_{30}, A_{60}, S_{30}, S_{60}\right)$.

\subsection{2. $\quad$ Parameters values and inputs}

Model parameters were fit to physical values observed at the studied site:

- a shifted exponential distribution of the headways with minimum headway $t_{m}=2 \mathrm{~s}$, 
- an impeding flow corresponding to the circulating flow without including a proportion of the exiting flow,

- $t_{f}=3 \mathrm{~s}$,

- $\quad \mu=0.33$ (see Chevallier and Leclercq, 2007),

- $\quad \gamma$ is not used since there is no congestion in the circulatory roadway,

- $\quad \kappa_{A}=\kappa_{D}=\kappa_{I}=\kappa_{C}=0.21 \mathrm{veh} / \mathrm{m}$,

- $u_{A}=u_{D}=12.4 \mathrm{~m} / \mathrm{s}$ and $u_{I}=u_{C}=5.3 \mathrm{~m} / \mathrm{s}$,

- $w_{A}=w_{D}=w_{I}=w_{C}=4.17 \mathrm{~m} / \mathrm{s}$.

Inputs were calculated as follows

- $\quad \Lambda_{i}(t)$ are aggregated flows over 90s-intervals calculated from vehicle counts at $A_{60}, A_{2}, A_{3}$ and $A_{4}$,

- $\Omega_{j}(t)=\frac{1}{t_{m}} \forall j \in[1, d]$ (no congestion in the vicinity of the roundabout),

- $\quad \chi_{i j}(t)$ are aggregated coefficients over 90s-intervals calculated from vehicle ID and vehicle counts at each entry $i$ and exit $j$.

\subsubsection{Simulation results}

In the multi-destination model, all vehicles drive around the central island only once. Hence, the model does not account for extra turns when drivers hesitate in choosing their exit. In the remaining, all observed extra movements were released from the vehicle counts database (about $1 \%$ of total counts). Simulated and observed $N$-curves during the morning period are shown in figure 8 for nine different locations. Curves are depicted in an oblique coordinate system to magnify their vertical displacements expressed in vehicles (see Munoz and Daganzo, 2002). 

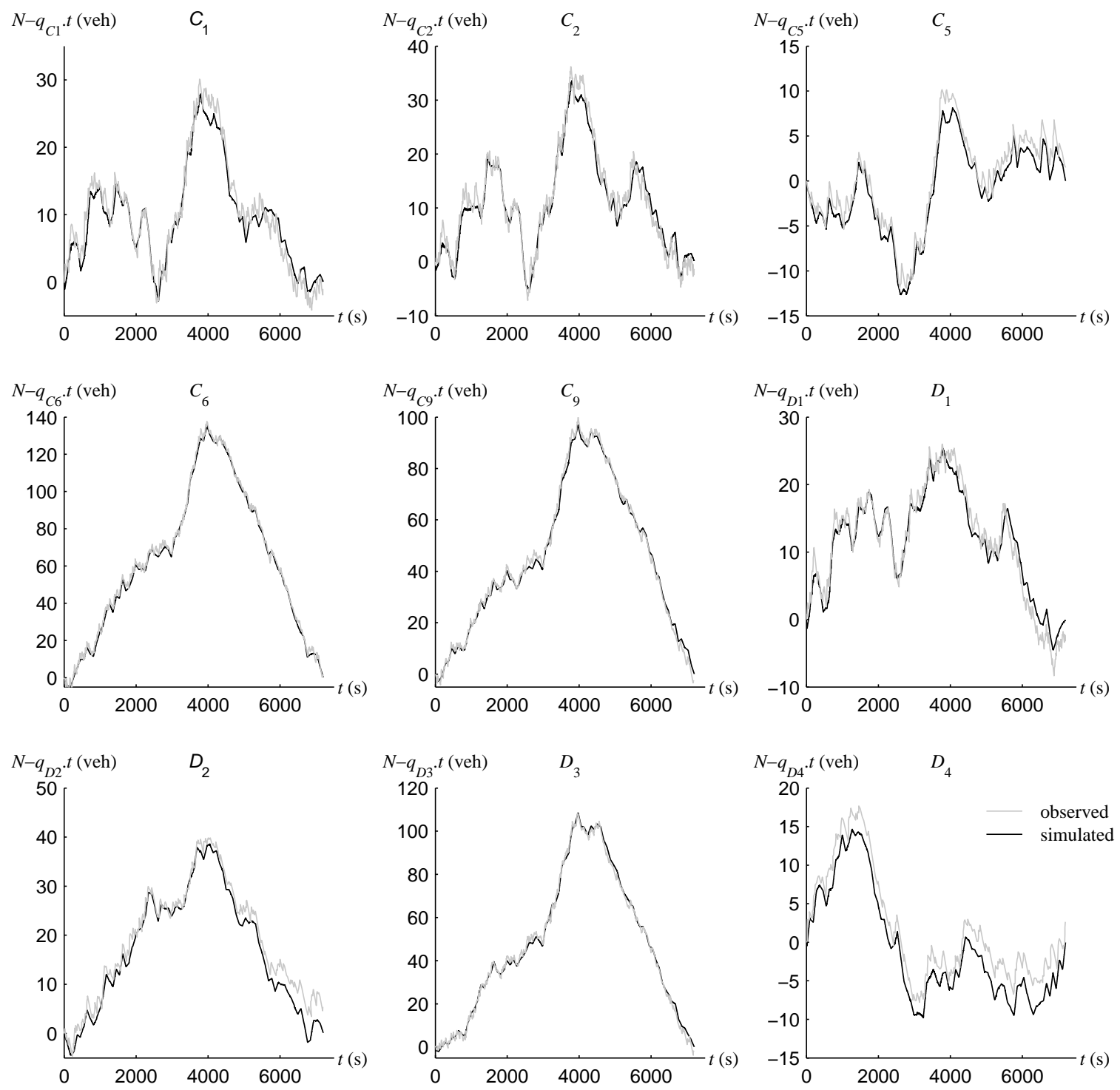

Fig.8. Consistency of the overall model with O-D patterns at different locations

The discrepancy between curves never exceeds 5 vehicles $(0.2 \%$ of the total entering vehicles). Note that similar conclusions can be drawn for the evening period. These results show evidence of the good general behaviour of the roundabout model and particularly its relevance with the O-D patterns of entry flows. 


\subsection{Merge model validation}

\subsubsection{Data collection}

To complete the model validation, it is worth studying in more details the merge model which plays a crucial role in the overall algorithm. The flow allocation scheme in uncongested regime is the main contribution of the merge model. Hence, we will study the relevance of simulated entering flows with empirical data when the circulatory roadway is uncongested. Data were collected at the conflict zone illustrated in figure $7 \mathrm{~b}$ during a 30mn-period. People with pocket laptop computers were in charge to record vehicle number plates and passing times $1200 \mathrm{~m}$ upstream the yield-line (position $A$ ) and at the entry (position $B$ ). Again, the combination of video tapes with the AUTOSCOPE software was used to extract circulating and exit vehicle counts at locations $R$ (right lane), $L$ (left lane) and $D$ (departure).

\subsubsection{Computation of $\overline{q_{I}}$ for the traffic signal timing}

Although the conflict zone has only one circulating lane as explained above, in practice, a non-zero, yet moderated flow is observed at $R$ (representing $17 \%$ of the total circulating flow). The issue of how computing the mean impeding flow $\overline{q_{I}}$ used in the signal timing calculation should therefore be raised.

As expressed in Hagring (2000), circulating vehicles of both lanes should be considered as impeding traffic, the strength of the impediment being expressed by different sizes of the critical gap according to the target lane. The critical gap is generally larger for the right lane (between 4.3 and 4.6s) than for the left lane (between $4 \mathrm{~s}$ and $4.4 \mathrm{~s}$ ). For simplification, we neglect this aspect by taking the same critical gap of $4.45 \mathrm{~s}$ for both lanes. From Hagring's work (1998) we can derive the global cumulative headway distribution for a two-lane road when headways on both lanes follow a shifted exponential distribution with a 
common $t_{m}$ and mean flows $q_{R}$ (right lane) and $q_{L}$ (left lane). With the observed unbalanced flows at $R$ and $L$, this distribution is very close to a shifted exponential distribution with parameters $t_{m}$ and mean flow $q_{R}+q_{L}$. Hence, the circulatory roadway can be modeled as a one-lane link in which the flow is $q_{R}+q_{L}$.

Moreover, several studies (Hagring, 2001) (Mereszczak et al., 2006) have demonstrated the need to compute the impeding flow as the sum of the circulating flow and a proportion of the exiting flow. For two-way-stop-controlled intersections Kyte et al. (1996) concluded that the capacity prediction improved by including at least $50 \%$ of exiting vehicles. We chose a proportion of $60 \%$ in our study to finally compute $\overline{q_{I}}$ :

$$
\overline{q_{I}}=\underset{90 s}{\operatorname{mean}}\left[q_{R}+q_{L}+0.6 q_{D}\right]
$$

\subsection{3. $\quad$ Parameters values and inputs}

The parameters values were calibrated as follows:

- a shifted exponential distribution for the headways with location parameter $t_{m}=2 \mathrm{~s}$ and impeding flow $q_{R}+q_{L}+0.6 q_{D}$

- $t_{f}=2.45 \mathrm{~s}$,

- $\quad \mu=0.33$,

- $\quad \gamma$ is not used since there is no congestion in the circulating roadway,

- $\quad \kappa_{A}=\kappa_{D}=0.18 \mathrm{veh} / \mathrm{m}$ and $\kappa_{l}=\kappa_{C}=0.36 \mathrm{veh} / \mathrm{m}$,

- $u_{A}=u_{D}=18.8 \mathrm{~m} / \mathrm{s}$ and $u_{I}=u_{C}=10 \mathrm{~m} / \mathrm{s}$,

- $w_{A}=w_{D}=3.26 \mathrm{~m} / \mathrm{s}$ and $w_{I}=w_{C}=3.84 \mathrm{~m} / \mathrm{s}$.

The approach leg was directly fed by vehicle counts at $A$. The downstream supply of the departure and circulating links was fixed to $1 / t_{m}$ (no congestion). 


\subsubsection{Simulation results}

In figure 9 a we compare the simulated $N$-curve with the observed $N$-curve at position $B$ in an oblique coordinate system. One can notice that the simulated entering flow correctly fits the experimental data. The maximum discrepancy between the $N$-curves is about 5 vehicles (over 258 observed).

Observed and simulated average delays on the last $1200 \mathrm{~m}$ of the approach leg are depicted in figure $9 \mathrm{~b}$ for an aggregation period of $5 \mathrm{mn}$. Simulated delays accurately follow the time evolution of observations. Notice that, as the average queue length over a gapblock cycle is obtained from the product of the average delay and the arriving flow rate, the model also gives accurate average queue length estimates. During the first 15 minutes of the measurement period, flows are relatively high. The simulated average delay is closed to the observed one (17.9s against 17.2 s, i.e. a $4.3 \%$ overestimation). For the remaining $15 \mathrm{mn}$ period, traffic intensity decreases on both approach and circulating roads. The simulated average delay drops to $9.1 \mathrm{~s}$ against $10.8 \mathrm{~s}$ that is to say a $16 \%$ underestimation. This underestimation of delays can be explained by the fact that the model calculates the fictive signal timing independently of the arrivals on the approach leg. However, worse-off traffic situations in which approaching and circulating platoons arrive simultaneously can occur (Chevallier and Leclercq, 2006). This phenomenon is not necessary reproduced in the model if the traffic light is green when the approaching flow is high. It is all the more sensitive as the traffic intensity is low. Moreover the impacts of the continuous assumption in traffic flow modelling could be pointed out. Instead of delaying an entire number of vehicles, the red colour signal may only affect a non-integer part of them, thus reducing the delays. Anyway, it should be highlighted that a slight underestimation of delays when traffic intensity is low does not really matter since practitioners worry about roundabout performances under higher traffic flows. For this practical need, our model seems very 
efficient compared to classical macroscopic merge models which do not predict any delay when the entry demand is lower than the merging capacity.
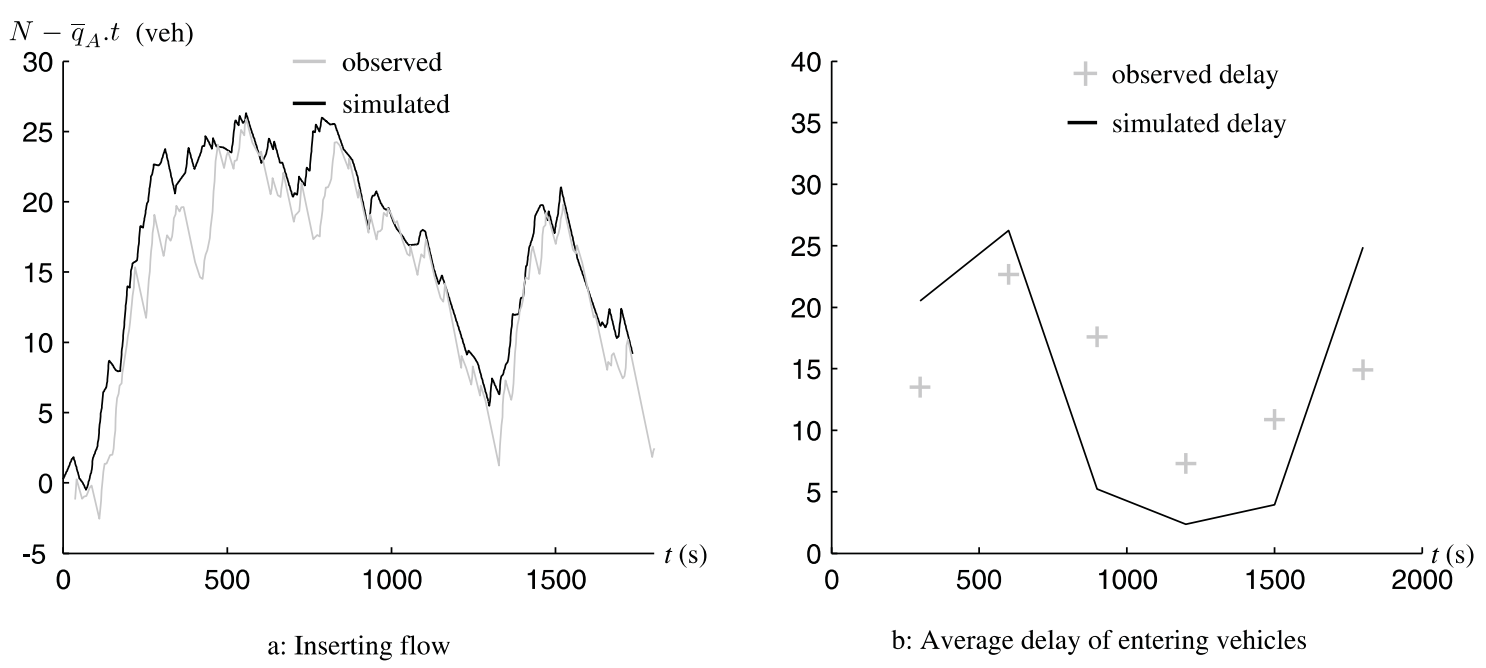

Fig.9. Merge model validation

\section{CONCLUSION}

To fill the shortage of parsimonious simulation tools, a dynamic macroscopic model for single-lane roundabouts was proposed. New specific algorithms were developed to overcome the main limitations of classical combination of aggregated merge, diverge and link models. Particularly, the model is able (i) to capture the effects of O-D patterns of entry flows thanks to a multi-destination formulation and (ii) to simulate the delays and queues triggered by the give way rule at the entries thanks to a fictive traffic light. It was shown that the simulated results for entry, circulating and exit flows were consistent with empirical data collected at two different roundabouts and at different day-time periods. As a result, the roundabout model can be fully integrated into a traffic flow simulation package to assess the local impacts of transport or environmental policies.

Further research should be conducted to check the sensitivity of the merge parameters to geometric layout, especially the priority ratios and the amount of exiting vehicles in the 
impeding traffic. Additional experimental data should be collected to complete the model validation in terms of average delays but also in terms of other traffic performance variables like the geometric delay, the back of the queue location or some percentile of the queue length distribution. It could also be worth extending this model to multi-lane roundabouts. For this, two criteria should be accounted for: (i) modelling the insertion process in a lane-by-lane framework as in the aaSIDRA model thanks to one fictive traffic signal per lane and (ii) accounting for lane changing (Laval and Daganzo, 2006) and for the effects of unequal lane utilisation.

\section{ACKNOLEDGEMENTS}

The authors are grateful to Catherine Barthe (ZELT) for her valuable help in the collection and processing of the Toulouse data, to the Centre for the Study of Urban Planning, Transport and Public Facilities (CERTU) for funding the Toulouse experimental study, as well as to Christine Buisson for kindly providing the Lyon data. The authors would also greatly thank all the reviewers for their relevant comments.

\section{REFERENCES}

Akçelik, R., Besley, M., 2001. Microsimulation and analytical methods for modelling urban traffic. Proceedings of the Conference on Advance Modeling Techniques and Quality of Service in Highway Capacity Analysis, Truckee, California, USA.

Akçelik, R., Besley, M., 2004. Differences between the AUSTROADS roundabout guide and the aaSIDRA roundabout analysis approach. Proceedings of the $26^{\text {th }}$ Conference of Australian Institutes of Transport Research, Clayton, Melbourne.

Akçelik, R., 2005. Roundabout model calibration issues and a case study. Proceedings of Transportation Research Board National roundabout conference, Vail, Colorado, USA.

Buisson, C., Lebacque, J.P., Lesort, J.B., 1996. STRADA, a discretized macroscopic model of vehicular traffic flow in complex networks based on the Godunov scheme. Proceedings of the CESA'96 IMACS Multiconference, Computational Engineering in Systems Applications, 976-981.

Cassidy, M.J., Ahn, S., 2005. Driver turn-taking behaviour in congested freeway merges. Transportation Research Record, 1934, 140-147. 
Chevallier, E., Leclercq, L., 2007. A macroscopic theory for unsignalized intersections, Transportation Research Part B, doi:10.1016/j.trb.2007.05.003.

Chevallier, E., Leclercq, L., 2006. Stochastic delay at traffic signals: influence of demand patterns. Proceedings of the EWGT Conference, Bari, Italy, 630-638.

Daganzo, C.F., 1995. The cell transmission model, Part II: Network traffic. Transportation Research Part B, 29 (2), 79-93.

Daganzo, C.F., 2005a. A variational formulation of kinematic waves: basic theory and complex boundary conditions. Transportation Research Part B, 39 (2), 187-196.

Daganzo, C.F., 2005b. A variational formulation of kinematic waves: Solution methods. Transportation Research Part B, 39 (10), 934-950.

Hagring, O., 2001. Derivation of capacity equation for roundabout entry with mixed circulating and exiting flows. Transportation Research Record, 1776, 91-99.

Hagring, O., 2000. Effects of OD flows on roundabout entry capacity. Proceedings of the 4th International Symposium on Highway Capacity, Maui, Hawaii.

Hagring, O., 1998. A further generalization of Tanner's formula. Transportation Research Part B, 32 (6), 423-429.

Highway Capacity Manual, 2006. Proposed Draft Chapter 17, Part C: Roundabouts. Proceedings of the AHB40 committee, Transportation Research Board, Washington, DC.

Jin, W.L., Zhang, H.M., 2003. On the distribution schemes for determining flows through a merge. Transportation Research Part B, 37 (6), 521-540.

Kimber, R.M., 1980. The Traffic Capacity of Roundabouts. TRRL Laboratory Report 942, Transport and Road Research Laboratory, Crowthorne, Berkshire, UK.

Kyte, M., Tian, Z., Mir, Z., Hameedmansoor, Z., Kittelson, W., Vandehey, M., Robinson, B., Brilon, W., Bondzio, L., Wu, N., Troutbeck, R., 1996. Capacity and level of service at unsignalized intersections, Final Report : Volume 1 - Two-Way-Stop-Controlled intersections, National Cooperative Highway Research Program 3-46, Transportation Research Board, Washington, DC.

Laval, J.A., Daganzo, C.F., 2006. Lane-changing in traffic streams. Transportation Research Part B, 40 (3), 251-264.

Lebacque, J.P., 1996. The Godunov scheme and what it means for the first order traffic flow models. In: Lesort, J.B. (Ed.), Proceedings of the 13th ISTTT, 647-678.

Lebacque, J.P., Khoshyaran, M.M., 2005. First order macroscopic traffic flow models: intersections modeling, network modeling. In: Mahmassani, H.S. (Ed.), Proceedings of the 16th ISTTT, 365-386.

Mereszczak, Y., Dixon, M., Kyte, M., Rodegerdts, L., Blogg, M., 2006. Including exiting vehicles in capacity estimation at single-lane U.S. roundabouts. Proceedings of the Transportation Research Board, Washington, DC.

Munoz, J.C., Daganzo, C.F., 2002. Fingerprinting traffic from static freeway sensors. Cooperative Transportation Dynamics, 1, 1-11.

Newell, G.F., 1993a. A simplified theory of kinematic waves in highway traffic, Part I: general theory. Transportation Research B, 27 (4), 281-287. 
Newell, G.F., 1993b. A simplified theory of kinematic waves in highway traffic, Part III: multi-destination flows. Transportation Research B, 27 (4), 305-313.

Robinson, B.W., 2000. Roundabout: an informational guide, Chapter 8: System consideration. Publication No. FHWA-RD-00-067.

Troutbeck, R.J., Kako, S., 1999. Limited priority merge at unsignalized intersections. Transportation Research Part A, 33 (3-4), 291-304.

Wu, N., 2001. A universal procedure for capacity determination at unsignalized (priority-controlled) intersections. Transportation Research Part B, 35 (6), 593-623. 